\title{
Correction of Thin Shell Finite Element Magnetic Models via a Subproblem Method
}

\author{
Patrick Dular $^{1,2}$, Vuong Q. Dang ${ }^{1}$, Ruth V. Sabariego ${ }^{1}$, Laurent Krähenbühl ${ }^{3}$, and Christophe Geuzaine ${ }^{1}$ \\ ${ }^{1}$ Department of Electrical Engineering and Computer Science, ACE, University of Liège, B-4000 Liège, Belgium \\ ${ }^{2}$ F.R.S.-FNRS, Fonds de la Recherche Scientifique, B-1000 Brussels, Belgium \\ ${ }^{3}$ Ampère (CNRS UMR5005), École Centrale de Lyon, Université de Lyon, F-69134 Écully Cedex, France
}

\begin{abstract}
A subproblem finite-element method is developed for correcting the inaccuracies near edges and corners inherent to thin shell models, for both magnetostatic and magnetodynamic problems. A thin shell solution, supported by a simplified mesh near the thin structures, serves as a source of a correction problem with the actual volumic thin regions alone in a homogeneous medium, concentrating the meshing effort on the thin regions only. Improvements of local fields are efficiently achieved and allow accurate force and loss calculations.
\end{abstract}

Index Terms_Finite-element method (FEM), model refinement, subdomain method, thin shell.

\section{INTRODUCTION}

$\mathbf{T}$ HIN SHELL (TS) finite-element (FE) models are commonly used to avoid meshing the thin structures and lighten the mesh of the surrounding regions [1]-[4]. Indeed, the fields in the thin regions are approximated by a priori known 1-D analytical distributions (throughout the shell thickness), that generally neglect end and curvature effects. Their interior is thus not meshed and is rather extracted from the studied domain, being reduced to a zero-thickness double layer with interface conditions (IC) linked to the inner analytical distributions [4]. The TS models suffer from inaccuracies in the vicinity of geometrical discontinuities, edges and corners, increasing with the thickness, which limits their range of validity.

The aim of this contribution is to develop a method to correct such inaccuracies for both magnetostatic and magnetodynamic problems, in particular for the magnetic vector potential formulations. The method is developed in the framework of the subproblem method (SPM), defining a complete solution as a sum of subproblem solutions, already applied to numerous corrections and problem splittings [5]-[10]. It can then be inspired and benefit from the SPM existing tools, adding its particularities.

\section{Thin SHell CorRection IN A SubProblem Method}

\section{A. Canonical Magnetodynamic/Static Problem}

A canonical magnetodynamic/static problem $p$, to be solved at step $p$ of the SPM, is defined in a domain $\Omega_{p}$, with boundary $\partial \Omega_{p}=\Gamma_{p}=\Gamma_{h, p} \cup \Gamma_{b, p}$. The eddy current conducting part of $\Omega_{p}$ is denoted $\Omega_{c, p}$ and the non-conducting one $\Omega_{c, p}^{C}$, with $\Omega_{p}=$ $\Omega_{c, p} \cup \Omega_{c, p}^{C}$. Massive inductors belong to $\Omega_{c, p}$, whereas stranded inductors belong to $\Omega_{c, p}^{C}$. Subscript $p$ refers to the associated

Manuscript received May 31, 2010; accepted August 30, 2010. Date of current version April 22, 2011. Corresponding author: P. Dular (e-mail: patrick. dular@ulg.ac.be). problem $p$. The equations, material relations and boundary conditions (BCs) of problem $p$ are

$$
\begin{aligned}
\operatorname{curl} \boldsymbol{h}_{p} & =\boldsymbol{j}_{p} \\
\operatorname{div} \boldsymbol{b}_{p} & =0 \\
\operatorname{curl} \boldsymbol{e}_{p} & =-\partial_{t} \boldsymbol{b}_{p} \\
\boldsymbol{h}_{p} & =\mu_{p}^{-1} \boldsymbol{b}_{p}+\boldsymbol{h}_{s, p} \\
\boldsymbol{j}_{p} & =\sigma_{p} \boldsymbol{e}_{p}+\boldsymbol{j}_{s, p} \\
\boldsymbol{n} \times\left.\boldsymbol{h}_{p}\right|_{\Gamma_{h, p}} & =\boldsymbol{j}_{f, p} \\
\left.\boldsymbol{n} \cdot \boldsymbol{b}_{p}\right|_{\Gamma_{b, p}} & =\boldsymbol{f}_{f, p} \\
\boldsymbol{n} \times\left.\boldsymbol{e}_{p}\right|_{\Gamma_{e, p} \subset \Gamma_{b, p}} & =\boldsymbol{k}_{f, p}
\end{aligned}
$$

where $\boldsymbol{h}_{p}$ is the magnetic field, $\boldsymbol{b}_{p}$ is the magnetic flux density, $\boldsymbol{e}_{p}$ is the electric field, $\boldsymbol{j}_{p}$ is the electric current density, $\mu_{p}$ is the magnetic permeability, $\sigma_{p}$ is the electric conductivity and $\boldsymbol{n}$ is the unit normal exterior to $\Omega_{p}$. Note that (1c) is only defined in $\Omega_{c, p}$ (as well as $\boldsymbol{e}_{p}$ ), whereas it is reduced to the form (1b) in $\Omega_{c, p}^{C}$. It is thus absent from the magnetostatic version of problem $p$. Further (3c) is more restrictive than (3b) in their homogeneous forms. Equations (1b-c) are fulfilled via the definition of a magnetic vector potential $a_{p}$ and an electric scalar potential $v_{p}$, leading to the $\boldsymbol{a}_{p}$-formulation, with

$$
\begin{aligned}
\operatorname{curl} \boldsymbol{a}_{p} & =\boldsymbol{b}_{p} \\
\boldsymbol{\boldsymbol { e } _ { p }} & =-\partial_{\boldsymbol{t}} \boldsymbol{a}_{p}-\operatorname{grad} v_{p} \\
\boldsymbol{n} \times\left.\boldsymbol{a}_{p}\right|_{\Gamma_{b, p}} & =\boldsymbol{a}_{f, p} .
\end{aligned}
$$

For various purposes, also for a TS representation, some paired portions of $\Gamma_{p}$ can define double layers, with the thin region in between exterior to $\Omega_{p}$ [5]-[9]. They are denoted $\gamma_{p}^{+}$ and $\gamma_{p}^{-}$and are geometrically defined as a single surface $\gamma_{p}$ with ICs, fixing the discontinuities $\left([\cdot]_{\gamma_{p}}=\left.\cdot\right|_{\gamma_{p}^{+}}-\left.\cdot\right|_{\gamma_{p}^{-}}\right)$

$$
\begin{aligned}
& {\left[\boldsymbol{n} \times \boldsymbol{h}_{p}\right]_{\gamma_{p}}} \\
& {\left[\boldsymbol{n} \cdot \boldsymbol{b}_{p}\right]_{\gamma_{p}}} \\
& {\left[\boldsymbol{n} \times \boldsymbol{e}_{p}\right]_{\gamma_{p}}} \\
& {\left[\boldsymbol{n} \times \boldsymbol{a}_{p}\right]_{\gamma_{p}} .}
\end{aligned}
$$


With the definitions $\left(\left.\boldsymbol{n}_{\gamma_{p}} \equiv \boldsymbol{n}\right|_{\gamma_{p}}\right)=-\left(\left.\boldsymbol{n}^{+} \equiv \boldsymbol{n}\right|_{\gamma_{p}^{+}}\right)=$ $\left(\left.\boldsymbol{n}^{-} \equiv \boldsymbol{n}\right|_{\gamma_{p}^{-}}\right)$for the normal $\boldsymbol{n}$ in different contexts, one has, e.g., for $(5 a)$

$$
\begin{aligned}
{\left[\boldsymbol{n} \times \boldsymbol{h}_{p}\right]_{\gamma_{p}} } & =\boldsymbol{n}_{\gamma_{p}} \times\left.\boldsymbol{h}_{p}\right|_{\gamma_{p}^{+}}-\boldsymbol{n}_{\gamma_{p}} \times\left.\boldsymbol{h}_{p}\right|_{\gamma_{p}^{-}} \\
& =-\left(\boldsymbol{n}^{+} \times\left.\boldsymbol{h}_{p}\right|_{\gamma_{p}^{+}}-\boldsymbol{n}^{-} \times\left.\boldsymbol{h}_{p}\right|_{\gamma_{p}^{-}}\right) .
\end{aligned}
$$

The fields $\boldsymbol{h}_{s, p}$ and $\boldsymbol{j}_{s, p}$ in (2a) and (2b) are volume sources (VSs). The source $\boldsymbol{h}_{s, p}$ is usually used for fixing a remnant field in magnetic materials. The source $\boldsymbol{j}_{s, p}$ fixes the current density in inductors. With the SPM, $\boldsymbol{h}_{s, p}$ is also used for expressing changes of permeability and $\boldsymbol{j}_{s, p}$ for changes of conductivity, or for adding portions of inductors [6]-[10]. For changes in a region, from $\mu_{q}$ and $\sigma_{q}$ for problem $q$ to $\mu_{p}$ and $\sigma_{p}$ for problem $p$, the associated VSs $\boldsymbol{h}_{s, p}$ and $\boldsymbol{j}_{s, p}$ are

$$
\begin{aligned}
\boldsymbol{h}_{s, p} & =\left(\mu_{p}^{-1}-\mu_{q}^{-1}\right) \boldsymbol{b}_{q} \\
\boldsymbol{j}_{s, p} & =\left(\sigma_{p}-\sigma_{q}\right) \boldsymbol{e}_{q}
\end{aligned}
$$

for the total fields to be related by the updated relations $h_{q}+$ $\boldsymbol{h}_{p}=\mu_{p}^{-1}\left(\boldsymbol{b}_{q}+\boldsymbol{b}_{p}\right)$ and $\boldsymbol{j}_{q}+\boldsymbol{j}_{p}=\sigma_{p}\left(\boldsymbol{e}_{q}+\boldsymbol{e}_{p}\right)$.

The surface fields $\boldsymbol{j}_{f, p}, \boldsymbol{f}_{f, p}$, and $\boldsymbol{k}_{f, p}$ in (3a)-(3c), and $\boldsymbol{a}_{f, p}$ in (4c), are generally zero for classical homogeneous BCs. The discontinuities (5a)-(5d) are also generally zero for common continuous field traces. If nonzero, they define possible surface sources (SSs) that account for particular phenomena occurring in the thin region between $\gamma_{p}^{+}$and $\gamma_{p}^{-}$[5]-[9]. This is the case when some field traces in a problem $q$ are forced to be discontinuous. The continuity has to be recovered after a correction via a problem $p$. The SSs in problem $p$ are thus to be fixed as the opposite of the trace solution of problem $q$.

Each problem $p$ is to be constrained via the so defined VSs and SSs from parts of solutions of other problems. This is a key element of the SPM, offering a wide variety of possible corrections, in particular of TS solutions as shown hereafter.

\section{B. Thin Shell Model}

A volumic shell $\Omega_{t s, p}$ initially in $\Omega_{c, p}$ or $\Omega_{c, p}^{C}$ is extracted from $\Omega_{p}$ and then considered with the double layer TS surface $\Gamma_{t s, p}$. The TS model [4] written with the $\boldsymbol{a}_{p}$-formulation, requires a free (unknown) discontinuity $\boldsymbol{a}_{d, t, p}$ of the tangential component $\boldsymbol{a}_{t, p}=\left(\boldsymbol{n} \times \boldsymbol{a}_{p}\right) \times \boldsymbol{n}$ of $\boldsymbol{a}_{p}$ through the TS, i.e.,

$$
\left[\mathbf{a}_{t, p}\right]_{\Gamma_{t s, p}}=\mathbf{a}_{d, t, p} \quad \text { or } \quad\left[\boldsymbol{n} \times \mathbf{a}_{t, p}\right]_{\Gamma_{t s, p}}=\boldsymbol{n} \times \boldsymbol{a}_{d, t, p}
$$

with a fixed zero value along the TS border $\partial \Gamma_{t s, p}$, which neglects the magnetic flux entering there. To explicitly express this discontinuity, one defines [4]

$$
\left.\boldsymbol{a}_{p}\right|_{\Gamma_{t s, p}^{+}}=\boldsymbol{a}_{c, p}+\boldsymbol{a}_{d, p},\left.\quad \boldsymbol{a}_{p}\right|_{\Gamma_{t s, p}^{-}}=\boldsymbol{a}_{c, p}
$$

with $\boldsymbol{a}_{c, p}$ the continuous component of $\boldsymbol{a}_{p}$. (9) also applies on $\Gamma_{t s, p}$ for the tangential components $\boldsymbol{a}_{t, p}, \boldsymbol{a}_{c, t, p}$, and $\boldsymbol{a}_{d, t, p}$.

The BCs and ICs associated with the TS, of impedance BC type [4], are then defined via $\boldsymbol{a}_{c, t, p}$ and $\boldsymbol{a}_{d, t, p}$ as

$$
\begin{aligned}
& {\left[\boldsymbol{n}_{t s} \times \boldsymbol{h}_{p}\right]_{\Gamma_{t s, p}}=-\sigma_{p} \beta_{p} \partial_{t}\left(2 \boldsymbol{a}_{c, t, p}+\boldsymbol{a}_{d, t, p}\right)} \\
& \boldsymbol{n}_{t s} \times\left.\boldsymbol{h}_{p}\right|_{\Gamma_{t s, p}^{+}} ^{+}+\boldsymbol{n}_{t s} \times\left.\boldsymbol{h}_{p}\right|_{\Gamma_{t s, p}^{-}}=-\left(\mu_{p} \beta_{p}\right)^{-1} \boldsymbol{a}_{d, t, p}
\end{aligned}
$$

$$
\begin{aligned}
& \beta_{p}=\gamma_{p}^{-1} \tanh \left(\gamma_{p} d_{p} / 2\right), \quad \gamma_{p}=(1+j) / \delta_{p}, \\
& \delta_{p}=\sqrt{2 /\left(\omega \sigma_{p} \mu_{p}\right)}
\end{aligned}
$$

where $d_{p}$ is the local TS thickness (possibly non-constant), $\delta_{p}$ is the skin depth in the TS, $\omega=2 \pi f$ with $f$ the frequency, $j$ is the imaginary unit and $\partial_{t} \equiv j \omega$. For $\beta_{p} \approx d_{p}$, one has $\beta_{p} \approx d_{p} / 2$. In statics, (10a) is zero and (10b) is $-2\left(\mu_{p} d_{p}\right)^{-1} \boldsymbol{a}_{d, t, p}$.

\section{Thin Shell Correction}

Once obtained, the TS solution, renamed solution $q$, is corrected by another solution $p$ that overcomes the TS assumptions. The SPM offers the tools to perform such a model refinement, thanks to simultaneous SSs and VSs. Indeed, SSs related to ICs (5a) and (5d) can compensate the TS discontinuities $\left[\boldsymbol{n} \times \boldsymbol{h}_{q}\right]_{\Gamma_{t s, q}}(10 \mathrm{a})$ and $\left[\boldsymbol{n} \cdot \boldsymbol{b}_{q}\right]_{\Gamma_{t s, q}}$ via $\left[\boldsymbol{n} \times \boldsymbol{a}_{q}\right]_{\Gamma_{t s, q}}$ (8) to suppress the TS representation, in parallel to VSs (7a) and (7b) in the added volumic shell that account for the associated $\mu_{p}$ and $\sigma_{p}$, different from these of problem $q$ that characterized the ambient region $\left(\mu_{q}=\mu_{0}\right.$ and $\left.\sigma_{q}=0\right)$.

\section{FE WEAK FORMULATIONS}

\section{A. Canonical b-Conform Weak Formulations}

The weak $\boldsymbol{a}_{p}$-formulation of the canonical problem $p$ is obtained from the weak form of the Ampère equation (1a), i.e., [6]-[9]

$$
\begin{aligned}
& \left(\mu_{p}^{-1} \operatorname{curl} \boldsymbol{a}_{p}, \operatorname{curl} \boldsymbol{a}^{\prime}\right)_{\Omega_{p}}+\left(\boldsymbol{h}_{s, p}, \operatorname{curl} \boldsymbol{a}^{\prime}\right)_{\Omega_{p}}-\left(\boldsymbol{j}_{s, p}, \boldsymbol{a}^{\prime}\right)_{\Omega_{p}} \\
& \quad+\left(\sigma_{p} \partial_{t} \boldsymbol{a}_{p}, \boldsymbol{a}^{\prime}\right)_{\Omega_{p}}+\left\langle\boldsymbol{n} \times \boldsymbol{h}_{s, p}, \boldsymbol{a}^{\prime}\right\rangle_{\Gamma_{h, p}}+\left\langle\boldsymbol{n} \times \boldsymbol{h}_{p}, \boldsymbol{a}^{\prime}\right\rangle_{\Gamma_{b, p}} \\
& +\left\langle-\left[\boldsymbol{n} \times \boldsymbol{h}_{p}\right]_{\gamma_{p}}, \boldsymbol{a}^{\prime}\right\rangle_{\gamma_{p}}=0, \quad \forall \boldsymbol{a}^{\prime} \in F_{p}^{1}\left(\Omega_{p}\right)
\end{aligned}
$$

where $F_{p}^{1}\left(\Omega_{p}\right)$ is a curl-conform function space defined on $\Omega_{p}$, gauged in $\Omega_{c, p}^{C}$, and containing the basis functions for $\boldsymbol{a}$ as well as for the test function $\boldsymbol{a}^{\prime}$ (at the discrete level, this space is defined by edge FEs; the gauge is based on the tree-co-tree technique); $(\cdot, \cdot)_{\Omega}$ and $\langle\cdot, \cdot\rangle_{\Gamma}$, respectively, denote a volume integral in $\Omega$ and a surface integral on $\Gamma$ of the product of their vector field arguments. The surface integral term on $\Gamma_{h, p}$ accounts for natural BCs of type (3a), usually zero. The term on the surface $\Gamma_{b, p}$ with essential BCs on $\boldsymbol{n} \cdot \boldsymbol{b}_{p}$ is usually omitted because it does not locally contribute to (12). It can be used for post-processing a solution, a part of which $\boldsymbol{n} \times\left.\boldsymbol{h}_{p}\right|_{\Gamma_{b, p}}$ having to act further as a SS [5]-[9].

At the discrete level, the characteristics of the required meshes for each problem $p$ in the SPM totally differ. For the TS problem, the mesh describes the details of the source and is simplified near the TS regions, whereas the correction problem mesh focuses on the actual volumic thin region, finely discretized in a homogeneous medium. The required sources for the correction problem have to be transferred from the TS mesh to the correction mesh. A rigorous expression of the sources is crucial for the efficiency of the method.

\section{B. Thin Shell Model}

The TS model is defined in (12) via a free discontinuity $\boldsymbol{a}_{d, t, p}$ along the TS and $\boldsymbol{a}_{d, p}$ in the exterior region adjacent to $\Gamma_{t s, p}^{+}$, as 
well as IC (10a) and a BC for $\boldsymbol{n}_{t s} \times\left.\boldsymbol{h}_{p}\right|_{\Gamma_{t s, p}^{+}}$obtained by adding (10a) and (10b). The explicit discontinuity is to be used as a test function in (12), with contributions in the volume integrals on $\Omega_{p}$ (limited to the FE layer on the positive side of the TS) and in the surface integral term on $\gamma_{p}=\Gamma_{t s, p}$, i.e.,

$$
\begin{aligned}
\left\langle-\left[\boldsymbol{n} \times \boldsymbol{h}_{p}\right]_{\Gamma_{t s, p}}, \boldsymbol{a}^{\prime}\right\rangle_{\Gamma_{t s, p}} & =\left\langle-\left[\boldsymbol{n}_{t s} \times \boldsymbol{h}_{p}\right]_{\Gamma_{t s, p}}, \boldsymbol{a}_{c, t, p}^{\prime}\right\rangle_{\Gamma_{t s, p}} \\
+ & \left\langle-\boldsymbol{n}_{t s} \times\left. h_{p}\right|_{\left.\Gamma_{t s, p}^{+}, \boldsymbol{a}_{d, t, p}^{\prime}\right\rangle_{\Gamma_{t s, p}}}\right.
\end{aligned}
$$

which is reduced to $\left\langle\left(\mu_{p} d_{p}\right)^{-1} \boldsymbol{a}_{d, t, p}, \boldsymbol{a}_{d, t, p}^{\prime}\right\rangle_{\Gamma_{t s, p}}$ in statics.

\section{Projections of Solutions Between Meshes}

Some parts of a previous solution $\boldsymbol{a}_{q}$ serve as sources in a subdomain $\Omega_{s, p} \subset \Omega_{p}$ of the current problem $p$. At the discrete level, this means that this source quantity $\boldsymbol{a}_{q}$ has to be expressed in the mesh of problem $p$, while initially given in the mesh of problem $q$. This can be done via a projection method [11] of its curl limited to $\Omega_{s, p}$, i.e.,

$$
\left(\operatorname{curl} \boldsymbol{a}_{q-p}, \operatorname{curl} \boldsymbol{a}^{\prime}\right)_{\Omega_{s, p}}=\left(\operatorname{curl} \boldsymbol{a}_{q}, \operatorname{curl} \boldsymbol{a}^{\prime}\right)_{\Omega_{s, p},}, \forall \boldsymbol{a}^{\prime} \in F_{p}^{1}\left(\Omega_{s, p}\right)
$$

where $F_{p}^{1}\left(\Omega_{s, p}\right)$ is a gauged curl-conform function space for the $p$-projected source $\boldsymbol{a}_{q-p}$ (the projection of $\boldsymbol{a}_{q}$ on mesh $p$ ) and the test function $\boldsymbol{a}^{\prime}$.

\section{TS Correction-VSs in the Actual Volumic Shell and SSs for Suppressing the TS Representation}

Changes from $\mu_{q}$ and $\sigma_{q}$ to $\mu_{p}$ and $\sigma_{p}$, that occur in the volumic shell, are taken into account in (12) via the volume integrals $\left(\boldsymbol{h}_{s, p}, \operatorname{curl} \boldsymbol{a}^{\prime}\right)_{\Omega_{p}}$ and $\left(\boldsymbol{j}_{s, p}, \boldsymbol{a}^{\prime}\right)_{\Omega_{p}}$. The VS $\boldsymbol{h}_{s, p}$ is given by (7a), with $\boldsymbol{b}_{q}=$ curl $\boldsymbol{a}_{q}$. To avoid any cancellation error on the reaction field $\boldsymbol{b}_{p}$ for high $\mu_{p}$, the robust procedure developed in [9] has to be applied, with an intermediate change to an infinite $\mu_{p}$. The VS $\boldsymbol{j}_{s, p}$ is given by (7b), generally reduced to $\boldsymbol{j}_{s, p}=\sigma_{p} \boldsymbol{e}_{q}=\sigma_{p}\left(-\partial_{t} \boldsymbol{a}_{q}-\operatorname{grad} v_{q}\right)$. Potential $v_{q}$ is unknown in $\Omega_{c, p}^{C}$, but its determination via an electric problem [10] prior to problem $p$ is not needed: relation (2b) is then $\boldsymbol{j}_{p}=$ $\sigma_{p}\left(\boldsymbol{e}_{p}+\boldsymbol{e}_{q}\right)=\sigma_{p}\left(-\partial_{t} \boldsymbol{a}_{p}-\operatorname{grad} v_{p}-\partial_{t} \boldsymbol{a}_{q}-\operatorname{grad} v_{q}\right)$, with the freedom to fix $v_{p}$ and $v_{q}$ to zero and let $\boldsymbol{a}_{p}$ to react alone to $\boldsymbol{a}_{q}$.

At the discrete level, the primal source quantity $\boldsymbol{a}_{q}$ is projected in the mesh $p$ via (14), with $\Omega_{s, p}$ limited to the volumic shell.

Simultaneously to the VSs, SSs have to suppress the TS discontinuities, with ICs (5a) and (5d) to be defined as

$$
\begin{aligned}
& {\left[\boldsymbol{n} \times \boldsymbol{h}_{p}\right]_{\Gamma_{t s, p}}=-\left[\boldsymbol{n} \times \boldsymbol{h}_{q}\right]_{\Gamma_{t s, p}}} \\
& {\left[\boldsymbol{n} \times \boldsymbol{a}_{p}\right]_{\Gamma_{t s, p}}=-\boldsymbol{n} \times\left.\boldsymbol{a}_{d, t, q}\right|_{\Gamma_{t s, p}}}
\end{aligned}
$$

respectively, in weak and strong senses, i.e., via a surface integral and in $F_{p}^{1}\left(\Omega_{p}\right)$. IC (15b) strongly fixes $\boldsymbol{a}_{d, t, p}=-\boldsymbol{a}_{d, t, q}$. IC (15a) is weakly expressed via the last integral in (12), with $\gamma_{p}=\Gamma_{t s, p}=\Gamma_{t s, q}$. The so involved trace $\left[\boldsymbol{n} \times \boldsymbol{h}_{q}\right]_{\Gamma_{t s, p}}$ can be naturally expressed via the other (volume) integrals in (12), i.e.,

$$
\begin{aligned}
\left\langle-\left[\boldsymbol{n} \times \boldsymbol{h}_{p}\right]_{\Gamma_{t s, p}}, \boldsymbol{a}^{\prime}\right\rangle_{\Gamma_{t s, p}} & =-\left\langle-\left[\boldsymbol{n} \times \boldsymbol{h}_{q}\right]_{\Gamma_{t s, p}}, \boldsymbol{a}^{\prime}\right\rangle_{\Gamma_{t s, q}} \\
& =\left(\mu_{q}^{-1} \operatorname{curl} \boldsymbol{a}_{q}, \operatorname{curl} \boldsymbol{a}^{\prime}\right)_{\Omega_{p}=\Omega_{q}} .
\end{aligned}
$$

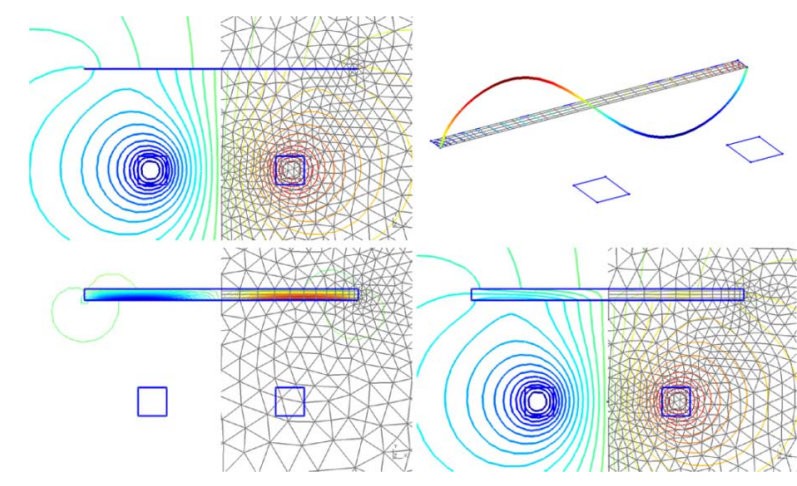

Fig. 1. Field lines for the magnetostatic TS model $\left(\boldsymbol{b}_{1}\right.$, top left $)$ and the correction solution $\left(\boldsymbol{b}_{2}\right.$, bottom left $)(d=5 \mathrm{~mm}$, plate length $120 \mathrm{~mm}$, relative permeability $\left.\mu_{r}=100\right)$ and the total solution $\left(\boldsymbol{b}_{1}+\boldsymbol{b}_{2}\right.$, bottom right $)$, with the different meshes used. Projection of TS solution $\left(a_{1}\right.$ in the volumic shell, elevation of $\boldsymbol{a}_{d, 1}$ norm along the TS surface, top right).

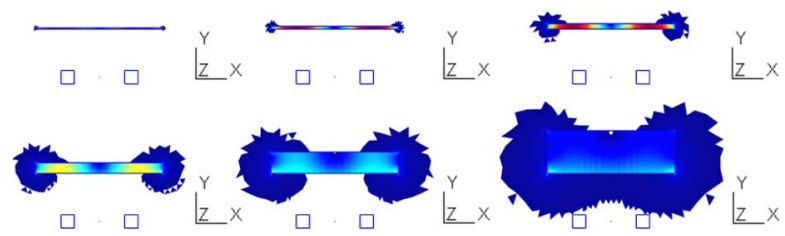

Fig. 2. Highlighted regions with a relative correction higher than $1 \%$ (in the plates and the vicinity of their ends) for $d=1.25,2.5,5,10,20$, and $40 \mathrm{~mm}$ (from left to right, top to bottom) (plate length $120 \mathrm{~mm}, \mu_{r}=100$ ).

The surface integral in (16) can also directly use (10a) at step $q$. At the discrete level, the volume integral in (16) is limited to the layers of FEs on both sides of $\Gamma_{t s, p}$, because it involves only the associated traces $\boldsymbol{n} \times\left.\boldsymbol{a}^{\prime}\right|_{\Gamma_{t s, p}}$. The source $\boldsymbol{a}_{q}$, including its discontinuity $\boldsymbol{a}_{d, q}$, initially in mesh $q$, has to be projected in mesh $p$ via (14), with $\Omega_{s, p}$ limited to the FE layers, which thus decreases the computational effort of the projection process. This also gives the SS for (15b).

\section{APPLICATION EXAMPLES}

The test problem consists of a plate located above an inductor (2-D model, Fig. 1). It is first considered via a TS FE magnetostatic model, with a lighter mesh (Fig. 1, top left). Then an SPM correction replaces the TS FEs with classical volumic FEs covering the plates and their neighborhood, with an adequate refined mesh, that does not include the inductor anymore (Fig. 1, bottom left). The projections of portions of TS solutions for the VS and SS are also illustrated (Fig. 1, top right). For each set of parameters, the SPM solution is compared with the classical solution calculated on a single mesh (Fig. 1, bottom right). The inaccuracies of the TS model, that increase with the plate thickness, particularly near the plate ends, are perfectly corrected whatever their order of magnitude (Fig. 2). The accuracy of the correction is directly linked to the volumic mesh of the plate and its neighboring. The correction process is then extended in magnetodynamics (see Fig. 3), leading to accurate corrections of magnetic flux and eddy current densities. Each correction is a reaction field that can influence the source solutions. Iterations between problems can thus be required [7], with a fast convergence due to the local nature of the reaction field. In the considered examples, the source problems do not need to be corrected because they only contain coils without any magnetic or conducting regions that would give their own reaction fields. The 


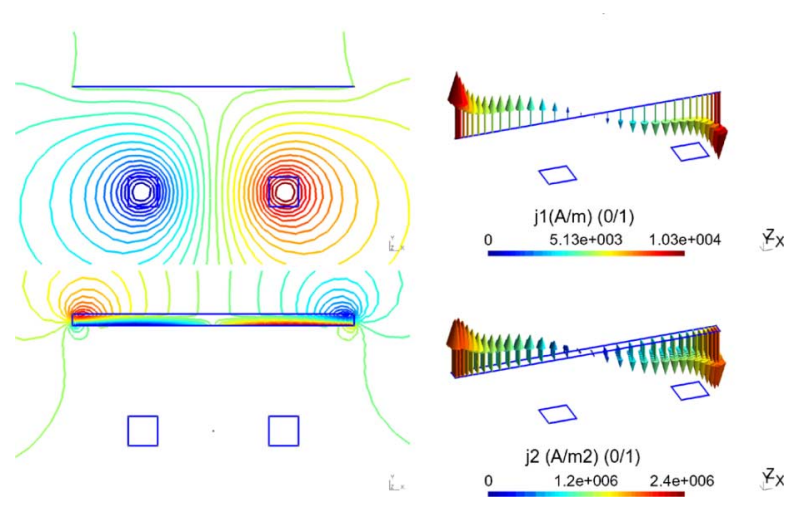

Fig. 3. Field lines (left) and eddy current density (right) for the magnetodynamic TS model $\left(\boldsymbol{b}_{1}, \boldsymbol{j}_{1}\right.$, top $)$ and the correction solution $\left(\boldsymbol{b}_{2}, \boldsymbol{j}_{2}\right.$, bottom $)(d=$ $5 \mathrm{~mm}$, plate length $\left.120 \mathrm{~mm}, \mu_{r}=1, \sigma=5.910^{7} \Omega^{-1} \mathrm{~m}^{-1}, f=300 \mathrm{~Hz}\right)$.

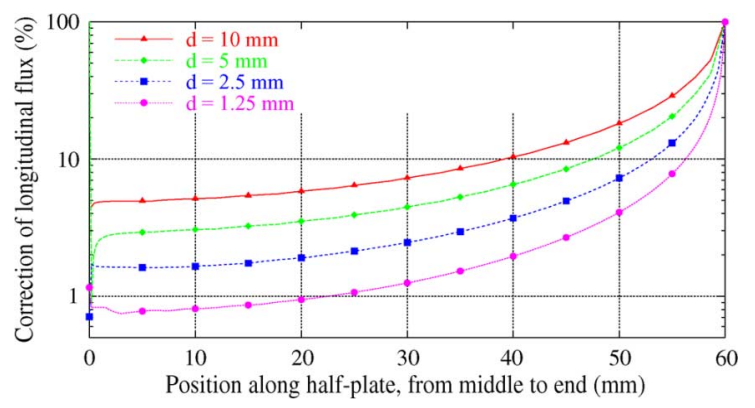

Fig. 4. Relative correction of the longitudinal magnetic flux along the plate for different plate thicknesses (plate length $120 \mathrm{~mm}, \mu_{r}=100$ ).

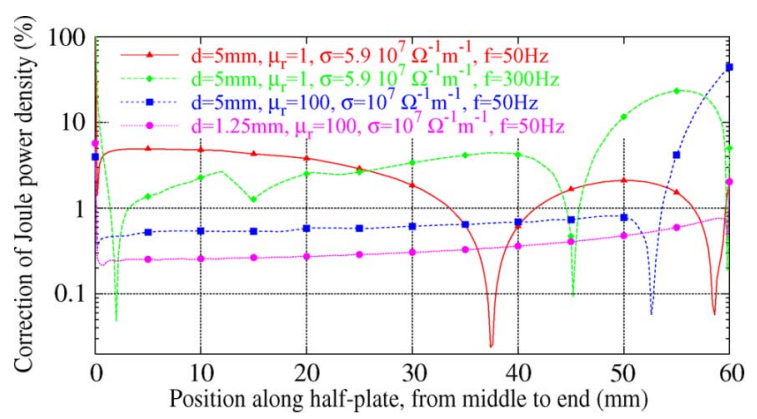

Fig. 5. Relative correction of the Joule power density along the plate, with effects of $d, \mu_{r}, \sigma$, and $f$.
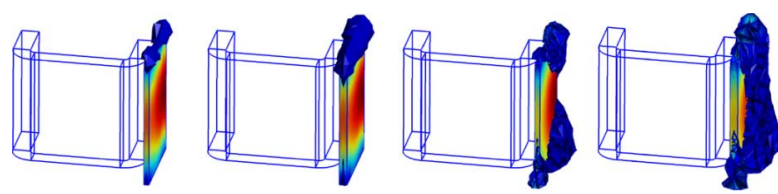

Fig. 6. TEAM problem 21 (1/4th of the geometry, magnetostatics): highlighted regions with a relative correction higher than $1 \%$ (in the plates and their neighboring) for $\left\{d=10 \mathrm{~mm}, \mu_{r}=100\right\},\left\{\mathrm{d}=15 \mathrm{~mm}, \mu_{r}=100\right\},\{d=$ $\left.10 \mathrm{~mm}, \mu_{r}=10\right\},\left\{d=15 \mathrm{~mm}, \mu_{r}=10\right\}$ (from left to right).

sum of the SPM solutions is thus equivalent to the complete solution, with an accuracy governed by the SPM meshes with particular refinements.

The corrections, usually of a few percents in the exterior region, can reach several tens of percents in the shells. For magnetic shells, the TS longitudinal magnetic flux is usually of poor quality near the shell ends (see Fig. 4), which is then perfectly corrected by the SPM correction. In magnetodynamics, a problem with a mainly longitudinal flux suffers from the same flux inaccuracies, whereas these will be lower with a transversal flux. The TS error on the eddy current density and the ensuing Joule power density depends on several parameters, as shown in Fig. 5. The inaccuracies on the Joule losses can reach 50\% in the end regions for some critical parameters: e.g., $45 \%$ with the $3 \mathrm{rd}$ set, with $\delta=2.3 \mathrm{~mm}$, or $30 \%$ with the 2 nd set, with $\delta=3.8 \mathrm{~mm}$, with $d=5 \mathrm{~mm}$ in both cases.

The correction scheme has been successfully applied in 3-D, for a particular configuration of TEAM problem 21 (coil and plate, Fig. 4), which opens it to further detailed studies.

\section{CONCLUSION}

The proposed correction scheme of TS models via a SPM leads to accurate field and current distributions in critical regions, the edges of plates, and so of the ensuing forces and Joule losses distributions. Even significant corrections can be obtained at a low computational cost thanks to the use of different adapted meshes, with simplifications in both TS and correction problems. The TS correction can constitute a particular step of the SPM developed in [6]-[9], which is particularly interesting in parametric analyses on geometrical and material parameters.

\section{ACKNOWLEDGMENT}

This work was supported by the F.R.S.-FNRS (Belgium), the Belgian Science Policy (IAP P6/21), and the Walloon Region.

\section{REFERENCES}

[1] L. Krähenbühl and D. Muller, "Thin layers in electrical engineering. Examples of shell models in analyzing eddy-currents by boundary and finite element methods," IEEE Trans. Magn., vol. 29, no. 2, pp. 1450-1455, Feb. 1993.

[2] C. Guerin, G. Tanneau, G. Meunier, X. Brunotte, and J. B. Albertini, "Three dimensional magnetostatic finite elements for gaps and iron shells using magnetic scalar potentials," IEEE Trans. Magn., vol. 30, no. 5, pp. 2885-2888, May 1994.

[3] Z. Ren, "Degenerated Whitney prism elements-general nodal and edge shell elements for field computation in thin structures," IEEE Trans. Magn., vol. 34, no. 5, pp. 2547-2550, May 1998.

[4] C. Geuzaine, P. Dular, and W. Legros, "Dual formulations for the modeling of thin electromagnetic shells using edge elements," IEEE Trans. Magn., vol. 36, no. 4, pp. 799-802, Apr. 2000.

[5] P. Dular, R. V. Sabariego, J. Gyselinck, and L. Krähenbühl, "Sub-domain finite element method for efficiently considering strong skin and proximity effects," in Proc. COMPEL, 2007, pp. 974-985.

[6] P. Dular, R. V. Sabariego, M. V. Ferreira da Luz, P. Kuo-Peng, and L. Krähenbühl, "Perturbation finite element method for magnetic model refinement of air gaps and leakage fluxes," IEEE Trans. Magn., vol. 45, no. 3, pp. 1400-1403, Mar. 2009.

[7] P. Dular, R. V. Sabariego, M. V. Ferreira da Luz, P. Kuo-Peng, and L. Krähenbühl, "Perturbation finite-element method for magnetic circuits," IET Sci., Meas. Technol., vol. 2, no. 6, pp. 440-446, 2008.

[8] P. Dular, R. V. Sabariego, and L. Krähenbühl, "Magnetic model refinement via a perturbation finite element method-From 1-D to 3-D," in Proc. COMPEL, 2009, pp. 974-988.

[9] P. Dular, R. V. Sabariego, C. Geuzaine, M. V. Ferreira da Luz, P. Kuo-Peng, and L. Krähenbühl, "Finite element magnetic models via a coupling of subproblems of lower dimensions," IEEE Trans. Magn., vol. 46, no. 8, pp. 2827-2830, Aug. 2010.

[10] P. Dular and R. V. Sabariego, "A perturbation method for computing field distortions due to conductive regions with h-conform magnetodynamic finite element formulations," IEEE Trans. Magn., vol. 43, no. 4, pp. 1293-1296, Apr. 2007.

[11] C. Geuzaine, B. Meys, F. Henrotte, P. Dular, and W. Legros, "A Galerkin projection method for mixed finite elements," IEEE Trans. Magn., vol. 35, no. 3, pp. 1438-1441, Mar. 1999. 